\title{
Clinical utilities of aromatase inhibitors in breast cancer
}

\author{
This article was published in the following Dove Press journal: \\ International Journal of Women's Health \\ 6 May 2015 \\ Number of times this article has been viewed
}

\author{
Saranya Chumsri \\ Department of Hematology \\ and Oncology, Mayo Clinic, \\ Jacksonville, FL, USA
}

Correspondence: Saranya Chumsri Department of Hematology and Oncology, Mayo Clinic, 4500 San Pablo Road South, Jacksonville, FL 32224, USA

Tel + I 9049536822

Fax + I 9049532315

Email chumsri.saranya@mayo.edu

\begin{abstract}
Aromatase is an enzyme that converts testosterones to estrogens. Inhibition of this enzyme has been shown to have several clinical utilities in breast cancer. Currently, there are three aromatase inhibitors (AIs) in clinical use, namely anastrozole, letrozole, and exemestane. AIs have been used in various clinical settings for breast cancer, ranging from chemoprevention in breast cancer to treating breast cancer in both early stage in the adjuvant setting and metastatic disease. This article reviews mechanism of action, AI classification, and clinical utilities of AIs in various clinical settings in the context of breast cancer.
\end{abstract}

Keywords: aromatase inhibitor, endocrine therapy, breast cancer

\section{Introduction}

Aromatase is an enzyme that is a member of the cytochrome P450 superfamily. Located on chromosome 15, aromatase is encoded in CYP19A1 gene. ${ }^{1,2}$ aromatase enzyme is involved in the last step of estrogen biosynthesis that converts testosterones to estrogens via aromatization process. Since aromatase has high specificity and is only involved in the last step steroid biosynthesis, inhibition of this enzyme does not affect levels of other biologically critical steroids. In postmenopausal women, aromatization of androgens, which are produced by the adrenal glands, serve as the main source of estrogen production after cessation of ovarian function. As the majority of breast cancers are hormone dependent, agents that target estrogen signaling pathway have been previously investigated as treatments for breast cancer. Tamoxifen was the first targeted therapy that inhibits estrogen signaling pathway. However, tamoxifen is associated with multiple concerning side effects, particularly risks of endometrial cancer and venous thromboembolism. ${ }^{3,4}$ This is the result of its partial estrogenic effects in the uterus and vascular system. Due to these unfavorable side effects and incomplete blockade of estrogen signaling pathways, an alternative approach to target the ligand production by inhibiting the aromatase enzyme was explored by Schwarzel et al in early $1970 \mathrm{~s} .{ }^{5,6}$ Currently, there are three aromatase inhibitors (AIs) in clinical use, namely anastrozole, letrozole, and exemestane. This review article summarizes classification of AIs as well as clinical utilities of AIs in various clinical settings in the context of breast cancer.

\section{Classification of Als}

AIs can be classified in two distinct subclasses, according to their chemical structures as shown in Table 1. An anti-epileptic drug, aminoglutethimide, was initially used for the treatment of breast cancer in the late 1970s. ${ }^{7}$ This drug suppresses multiple adrenal steroid productions by inhibiting various cytochrome P450 enzymes. Due to 
Table I Classification of Als

\begin{tabular}{lll}
\hline Generations & Type I steroidal inhibitors & Type 2 non-steroidal inhibitors \\
\hline $\begin{array}{l}\text { Nonspecific inhibitor } \\
\text { Previous selective inhibitors not }\end{array}$ & Formestane & Aminoglutethimide \\
currently in clinical use & & Fadrozole \\
& & Rogletimide \\
Vorozole & Anastrozole (Arimidex $\left.{ }^{\circledR}\right)$ & Letrozole (Femara $\left.^{\circledR}\right)$ \\
$\begin{array}{l}\text { Selective oral inhibitors currently } \\
\text { in clinical use }\end{array}$ & Exemestane (Aromasin $\left.{ }^{\circledR}\right)$ & \\
\hline
\end{tabular}

Note: Reprinted from The Journal of Steroid Biochemistry and Molecular Biology, Vol 125, Saranya Chumsri, Timothy Howes, Ting Bao, Gauri Sabnis, and Angela Brodie, Aromatase, aromatase inhibitors and Molecular Biology, pages 13-22., Copyright (20II), with permission from Elsevier. ${ }^{6}$

Abbreviation: Als, aromatase inhibitors.

inhibition of CYP11, cortisol replacement is required for this non-specific inhibitor. Later on, it was discovered that the key mechanism of action of aminoglutethimide is inhibition of aromatase enzyme. Given that this drug has to be given in combination with steroid replacement, aminoglutethimide is no longer in clinical use.

Subsequently, specific inhibitors of aromatase were developed. Mimicking the structure of androstenedione which is a substrate of aromatase enzyme, type I inhibitors or steroidal AIs bind to the substrate-binding site of the aromatase enzyme. Its reactive intermediate then binds covalently to the enzyme resulting in an irreversible inactivation of aromatase. These inhibitors are also known as "suicidal inhibitor" as the enzyme is inactivated by its own function. Steroidal AIs include formestane and exemestane. Exemestane is the only oral steroidal AI that is currently in clinical use since formestane requires painful intramuscular injections. In contrast, type II inhibitors or non-steroidal AIs bind non-covalently to the heme moiety of the aromatase enzyme and saturate the binding site. This type of AI causes reversible competitive inhibition of the enzyme. This subclass of AIs includes fadrozole, rogletimide, vorozole, anastrozole, and letrozole. Presently, only anastrozole and letrozole are in clinical use.

\section{Clinical utilities of Als in breast cancer}

Since the 1970s, it has been recognized that the majority of breast cancers are hormone dependent. Up to three quarters of breast cancers express hormone receptor, namely estrogen receptor (ER) and/or progesterone receptor. Modulations of either the receptors or its ligand have been proven to be effective strategies not only as treatments for breast cancer but also to prevent breast cancer. As described previously, AIs exert their activity by depleting estrogens which are the ligands that activate estrogen signaling pathways. In postmenopausal women, peripheral conversion of androgens to estrogens serves as the sole source of estrogen production after cessation of ovarian function. aromatase is present in multiple tissues, including adipose tissue, brain, blood vessels, skin, bone, normal breast tissue, and breast tumor cells. These locally produced estrogens in the breast tissue have an impact on tumor growth via paracrine or intracrine mechanisms. It has been shown previously that the levels of estrogens in breast tissue do not correlate with circulating estrogen levels in peripheral blood. ${ }^{8}$ While circulating estrogen levels are commonly low in postmenopausal women, several studies showed strikingly high levels of estrone, estrone sulfate, and estradiol in breast tumor tissue. ${ }^{9-11}$ Therefore, inhibition of aromatase results in further reduction of estrogens in circulation and local tissue in postmenopausal women.

Due to a large amount of aromatase substrate in the ovaries of premenopausal women and exquisite sensitivity of ovarian aromatase promoter to gonadotropins, AIs are not effective in reducing estrogen production in premenopausal women, unless concurrent ovarian suppression is administered. Inhibition of aromatase in premenopausal women initially results in transient reduction of estrogens, which activates hypothalamus and pituitary axis. This results in gonadotropin secretion that stimulates ovaries, causing subsequent rise in estrogen levels. ${ }^{12}$ Based on this observation, AIs have also been used in the stimulation protocols for ovulation induction in premenopausal breast cancer survivors to preserve fertility. After a short-term follow-up, the combination of $\mathrm{AI}$ and gonadotropins appeared to be safe in breast cancer patients and results in lower peak estradiol levels compared to standard in vitro fertilization. Currently, the combination of $\mathrm{AI}$ and gonadotropin is considered as a preferred ovarian induction protocol for women with breast cancer. ${ }^{13}$

Given the fact that AIs can stimulate ovaries via induction of gonadotropin secretion, the use of AIs in perimenopausal women can result in ovarian reactivation which can be detrimental in breast cancer patients. For premenopausal women who became amenorrheic after chemotherapy, Smith et a ${ }^{14}$ reported a cohort of 45 women who received adjuvant AIs after chemotherapy-induced amenorrhea of $\geq 6$ months with biochemical evidence of ovarian suppression. Alarmingly, 
$27 \%$ of women in this cohort resumed ovarian function; one woman became pregnant and ten women restarted menstrual period. Therefore, careful determination of menopausal status and serial hormone level monitoring, particularly follicle-stimulating hormone and estradiol levels, are critical to ensure the safe use for this class of drug.

\section{Metastatic setting}

Currently, AIs are commonly used as a first line treatment for postmenopausal women with metastatic hormone receptorpositive breast cancer. This is based on the results of several Phase III trials that demonstrated superiority of AIs compared to tamoxifen with regards to response rate, median time to progression, and clinical benefit rate. ${ }^{15-19}$ The largest trial in this setting enrolled 916 postmenopausal women and randomized patients to receive either tamoxifen or letrozole. This trial demonstrated a significant improvement in time to progression with letrozole compared to tamoxifen (42 versus [vs] 23 weeks, hazard ratio [HR] 0.70, $P=0.0001) .{ }^{20}$

More recently, the strategy to combine AIs with selective ER down regulator, fulvestrant, was investigated. This was based on the preclinical data that demonstrated more durable tumor control with complete blockade of ER signaling pathway, using AI in combination with fulvestrant compared to single agent $\mathrm{AI} .{ }^{21}$ These promising preclinical data have led to the conduction of two Phase III trials, the Fulvestrant and Anastrozole Combination Therapy (FACT) and Southwestern Oncology Group (SWOG) S226 trials. The FACT trial ${ }^{22}$ is a Phase III trial that randomized 514 postmenopausal women to receive either a single agent anastrozole or the combination of fulvestrant and anastrozole. Disappointingly, this trial showed no difference in median time to progression, 10.8 vs 10.2 months (HR 0.99, $P=0.91$ ) and overall survival (OS) was 37.8 vs 38.2 months (HR 1.0, $P=1.00$ ). In contrast, the SWOG S0226 trial, ${ }^{23}$ which enrolled a total of 707 patients and has a similar design, demonstrated not only a significant improvement in progression-free survival (13.5 vs 15 months, HR 0.8, $P=0.007$ ) but also OS benefit (41.3 vs 47.7 months, HR 0.81, $P=0.05$ ). Comparing the SWOG S0226 and FACT trials, there were more patients who presented with de novo metastasis without prior adjuvant endocrine therapy in the SWOG S0226 trial (32.2\% vs 59.7\%). Furthermore, a subset analysis in the SWOG S0226 trial showed that patients who did not receive prior endocrine therapy appeared to have more benefit from the combination of fulvestrant and anastrozole (HR 0.74, 95\% confidence interval [CI] 0.59-0.92 vs HR 0.89, CI 0.69-1.15). In addition, the Study of Faslodex Versus Exemestane With/Without Arimidex (SoFEA) trial, ${ }^{24}$ which evaluated the combination of fulvestrant and anastrozole vs fulvestrant alone or exemestane alone in patients with endocrine resistance, did not show the benefit of the combination over single agent fulvestrant or exemestane in this group of patients (median progression-free survival 4.4 vs 4.8 vs 3.4 months, respectively, $P=0.98$ ). Taken together, these data suggest that the combination of fulvestrant and AI may be beneficial in treatment of naïve patients who present with de novo metastasis. There appears to be no significant benefit of combining fulvestrant and AI in patients with endocrine resistance who previously received endocrine therapy.

\section{Adjuvant setting}

AIs are considered as standard of care for adjuvant treatment of postmenopausal women with hormone receptor-positive breast cancer. ${ }^{25}$ This is based on several Phase III clinical trials that compared the benefit of AIs to tamoxifen in the adjuvant setting. Several strategies were investigated in these Phase III trials, including upfront AIs, switching to AIs after 2-3 years of tamoxifen, and extended AI after completion of tamoxifen for 5 years. Across the board, AIs were shown to be superior to tamoxifen. ${ }^{6}$ For the upfront strategy, both anastrozole and letrozole for 5 years have been shown to significantly improve disease-free survival (DFS) compared to 5 years of tamoxifen in the Arimidex, Tamoxifen, Alone or in Combination $(\mathrm{ATAC})^{26}$ and Breast International Group (BIG) $1-98^{27}$ trials, respectively. However, there was no significant improvement in OS observed in these trials. The lack of OS benefit may be due to crossover in considerable numbers of patients after these trials were reported. Nevertheless, AIs appeared to be better tolerated with less treatment-related serious adverse events compared to tamoxifen (OR 0.57, $P<0.0001$ ). ${ }^{26}$ Of note, unlike the benefit seen with the combination of selective ER down regulator and AI, concurrent administration of anastrozole and tamoxifen in the ATAC trial showed detrimental effects in DFS compared to anastrozole single agent. ${ }^{26}$

For switching strategy, there were five clinical trials that compared 5 years of tamoxifen to sequential treatment of tamoxifen for 2-3 years followed by AIs. These trials include Austrian Breast and Colorectal Cancer Study Group (ABCSG)-8, BIG 1-98, ${ }^{27}$ German ARIMIDEX-Tamoxifen (ARNO 95), Italian Tamoxifen Anastrozole (ITA), and Intergroup Exemestane Study (IES) trials. ${ }^{28-30}$ All of these trials demonstrated significant improvement in DFS among patients who received sequential treatment compared to tamoxifen alone with the HR ranging from $0.57-0.76$. The BIG 1-98 trial was the only trial that directly compared the sequential treatment of AI followed by tamoxifen, the 
reverse sequence, and upfront treatment of AI or tamoxifen. There was no statistically significant difference between the two sequential arms compared to single agent letrozole. However, there were numerically more relapses within the first few years in tamoxifen followed by AI arm, particularly in patients with lymph node involvement. ${ }^{27}$ Taking these data together, this study suggests that high risk patients with axillary lymph node involvement should receive upfront AI. However, switching to tamoxifen after 2-3 years of AI can be considered in case of intolerability since there was no statistically significant difference in DFS among patients who received 5 years of AI compared to 2-3 years of AI followed by tamoxifen.

Since hormone receptor-positive breast cancer can recur several years after the diagnosis, the strategy for extended endocrine therapy with AIs after completion of tamoxifen for 5 years was evaluated in ABCSG-6a, ${ }^{31}$ MA.17, ${ }^{32}$ and National Surgical Adjuvant Breast and Bowel Project (NSABP) B-33. ${ }^{33}$ After completion of adjuvant tamoxifen for 5 years, these trials randomized patients to receive either 5 more years of AIs or placebo. All of these clinical trials also demonstrated statistically significant reduction in recurrence among patients receiving extended AIs with the HR ranging from $0.6-0.68$.

Invasive lobular carcinoma is a less common histologic subtype of breast cancer that accounts for approximately $10 \%-15 \%$ of breast cancer. ${ }^{34}$ More recently, unplanned subset analysis in the BIG 1-98 trial suggested that patients with invasive lobular carcinoma may derive more benefit with letrozole compared to tamoxifen. The HR for OS was 0.39 (95\% CI, 0.23-0.68) for letrozole in patients with invasive lobular carcinoma compared to OS HR of 0.7 (95\% CI, 0.57-0.86) for patients with invasive ductal carcinoma. Nonetheless, this observation was an unplanned subset analysis and future confirmation from other clinical trials will be needed to validate this finding.

More recently, the benefit of AIs in the adjuvant setting was extended to premenopausal women. Due to potential ovarian reactivation and subsequent increase in estrogen production in premenopausal women, AIs are only indicated in postmenopausal women. In premenopausal women, adequate suppression of estrogen production can be achieved when AIs are combined with ovarian suppression. ABCSG12 trial ${ }^{35}$ was the first trial that reported the results of ovarian suppression with goserelin in combination with either tamoxifen or anastrozole in premenopausal women. Disappointingly, this trial did not show a significant improvement in DFS among patients receiving goserelin in combination with anastrozole (HR 1.10, $P=0.59$ ). However, this trial was rather a small trial that enrolled 1,803 patients and the duration of adjuvant endocrine therapy was only 3 years, which is shorter than the current standard. Recently, the joint analysis of the two Phase III trials, the Suppression of Ovarian Function Trial (SOFT) and the Tamoxifen and Exemestane Trial (TEXT), ${ }^{36}$ reported opposite results. The combined analysis included a total of 4,690 premenopausal patients with hormone receptor positive breast cancer who were randomized to receive either ovarian suppression plus exemestane vs ovarian suppression plus tamoxifen for 5 years. With the median follow-up of 68 months, this combined analysis demonstrated a significant improvement in DFS in the exemestane group (91.1\% vs $87.3 \%$, HR 0.72, $P<0.001)$. However, this combined analysis did not address the question whether the combination is superior to a single agent tamoxifen and whether there is any additional benefit of ovarian suppression. More recently, subsequent analysis from the SOFT trial was reported to address these particular aspects. SOFT trial ${ }^{37}$ enrolled a total of 3,066 premenopausal women and randomized these patients to receive single agent tamoxifen, tamoxifen plus ovarian suppression, or exemestane plus ovarian suppression. Overall, this trial showed that an addition of ovarian suppression to tamoxifen did not provide a significant benefit over single agent tamoxifen (HR 0.83, $P=0.10$ ). However, the benefit of the combinations was more pronounced among patients with higher risks that warrant adjuvant chemotherapy. The rate of DFS at 5 years was $78 \%$ with tamoxifen alone, $82.5 \%$ with tamoxifen plus ovarian suppression, and $85.7 \%$ with exemestane plus ovarian suppression. The benefit is much more pronounced in women younger than 35 years of age. Among a total of 350 women younger than 35 years old, the rate of DFS at 5 years was $67.7 \%$ with tamoxifen alone, $78.9 \%$ with tamoxifen plus ovarian suppression, and $83.4 \%$ with exemestane plus ovarian suppression. Taking these results together, $\mathrm{AI}$ in combination with ovarian suppression represents the new standard of care option for the adjuvant treatment in premenopausal women with hormone receptor positive breast cancer, particularly patients with higher risk that warrant chemotherapy and younger patients less than 35 years of age. ${ }^{38,39}$

\section{Prevention setting}

In the past few years, two additional clinical trials demonstrated that AIs can also be used not only for the treatment of breast cancer but also to prevent breast cancer. As tamoxifen is associated with concerning side effects, particularly risks of endometrial cancer and venous thromboembolism, the use of tamoxifen in preventative setting has not been popularized. ${ }^{40,41}$ Given that AIs are not associated with these 
concerning side effects and the fact that AI is more effective than tamoxifen for the treatment of breast cancer, there has been a lot of interest in exploring this class of drug for chemoprevention in breast cancer.

Based on these rationales, AIs were evaluated as chemopreventative agents in two large Phase III trials, the National Cancer Institute of Canada Clinical Trials Group (NCIC CTG) MAP-3 trial and IBIS-II trial. The MAP-3 trial $^{42}$ is the first trial that was reported in 2011. This trial enrolled a total of 4,560 postmenopausal women who are older than 35 years of age with at least one of the following risk factors: 60 years of age or older, Gail 5-year risk score greater than 1.66\% (chances in 100 of invasive breast cancer developing within 5 years); prior atypical ductal hyperplasia atypical lobular hyperplasia, lobular carcinoma in situ, or ductal carcinoma in situ with mastectomy. Instead of direct comparison to tamoxifen, this trial randomized patients to receive either 5 years of exemestane or placebo. After a median follow-up of almost 3 years, there was statistically significantly less invasive breast cancer in the exemestane arm compared to the placebo (11 vs 32 cases, HR $0.35, P=0.002$ ). The results of this trial were supported by the subsequent report from the IBIS-II trial. ${ }^{43}$ This trial is also a Phase III trial that randomized postmenopausal women to receive either anastrozole or placebo. This trial included a total of 3,864 women with an increased risk of breast cancer (relative risk $\geq 4$ for women aged $40-44$ years, $\geq 2$ for women aged $45-60$ years, and $\geq 1.5$ for women aged 60-70 years), or any women with lobular carcinoma in situ, atypical ductal hyperplasia, atypical lobular hyperplasia, ductal carcinoma in situ within the last 6 months with completed adequate local therapy, or $>5 \%$ risk of breast cancer in 10 years based on the Tyrer-Cuzick model. After a median follow-up of 5 years, there was significantly less invasive breast cancer in the anastrozole arm compared to the placebo arm ( 32 vs 64 cases, HR $0.5, P=0.001$ ). With these results, AIs are currently considered as one of the chemoprevention options for postmenopausal women with high risk of breast cancer.

\section{Side effect profile}

In general, AIs are quite well-tolerated compared to cytotoxic chemotherapy. In contrast to tamoxifen, AIs are not associated with increased risk of thromboembolism and endometrial cancer. Common side effects of AIs include vasomotor symptoms, musculoskeletal symptoms, and bone loss. There are several medications that can help alleviate vasomotor symptoms. These medications include venlafaxine, gabapentin, and clonidine. ${ }^{44}$ Musculoskeletal symptoms have been reported in up to $50 \%$ of women taking AIs and up to $20 \%$ of these patients discontinue the treatment due to this side effect. ${ }^{45}$ In a small pilot study of 29 patients, duloxetine appeared to help alleviate these musculoskeletal symptoms. ${ }^{46}$ There is an ongoing Phase III trial evaluating duloxetine for treatment of AI-associated musculoskeletal symptoms (SWOG S1202). For bone loss, it is crucial to monitor bone density test yearly in women who take AIs. Currently, denosumab $\left(\right.$ Prolia $\left.^{\circledR}\right)$ is approved by the US Food and Drug Administration specifically for AI-induced bone loss.

\section{Conclusion}

AIs are a class of drug with broad clinical indications in breast cancer. These medications can be used in various spectrums of breast disease ranging from chemopreventative setting, adjuvant setting, to metastatic setting. In general, AIs are quite well tolerated with some unique side effects that can often be managed. These side effects include vasomotor symptoms, musculoskeletal symptoms, and bone loss. In particular, long-term use of AIs has been shown to be associated with risks of osteoporosis and fracture. Since these conditions are preventable, careful monitoring of bone density in patients taking AIs and early intervention is critical.

\section{Disclosure}

Dr Chumsri received research funding from Novartis.

\section{References}

1. Thompson EA Jr, Siiteri PK. Utilization of oxygen and reduced nicotinamide adenine dinucleotide phosphate by human placental microsomes during aromatization of androstenedione. J Biol Chem. 1974; 249(17):5364-5372.

2. Chen SA, Besman MJ, Sparkes RS, et al. Human aromatase: cDNA cloning, Southern blot analysis, and assignment of the gene to chromosome 15. DNA. 1988;7(1):27-38.

3. Fisher B, Costantino JP, Redmond CK, Fisher ER, Wickerham DL, Cronin WM. Endometrial cancer in tamoxifen-treated breast cancer patients: findings from the National Surgical Adjuvant Breast and Bowel Project (NSABP) B-14. J Natl Cancer Inst. 1994;86(7):527-537.

4. Jordan VC. Tamoxifen: toxicities and drug resistance during the treatment and prevention of breast cancer. Annu Rev Pharmacol Toxicol. 1995; 35:195-211.

5. Schwarzel WC, Kruggel WG, Brodie HJ. Studies on the mechanism of estrogen biosynthesis. 8 . The development of inhibitors of the enzyme system in human placenta. Endocrinology. 1973;92(3):866-880.

6. Chumsri S, Howes T, Bao T, Sabnis G, Brodie A. Aromatase, aromatase inhibitors, and breast cancer. J Steroid Biochem Mol Biol. 2011;125(1-2): $13-22$.

7. Santen RJ, Brodie H, Simpson ER, Siiteri PK, Brodie A. History of aromatase: saga of an important biological mediator and therapeutic target. Endocr Rev. 2009;30(4):343-375.

8. Bulun SE, Lin Z, Imir G, et al. Regulation of aromatase expression in estrogen-responsive breast and uterine disease: from bench to treatment. Pharmacol Rev. 2005;57(3):359-383.

9. van Landeghem AA, Poortman J, Nabuurs M, Thijssen JH. Endogenous concentration and subcellular distribution of estrogens in normal and malignant human breast tissue. Cancer Res. 1985;45(6): 2900-2906. 
10. Chetrite GS, Cortes-Prieto J, Philippe JC, Wright F, Pasqualini JR. Comparison of estrogen concentrations, estrone sulfatase and aromatase activities in normal, and in cancerous, human breast tissues. J Steroid Biochem Mol Biol. 2000;72(1-2):23-27.

11. Geisler J, Berntsen H, Lonning PE. A novel HPLC-RIA method for the simultaneous detection of estrone, estradiol and estrone sulphate levels in breast cancer tissue. J Steroid Biochem Mol Biol. 2000;72(5): 259-264.

12. Fabian CJ. The what, why and how of aromatase inhibitors: hormonal agents for treatment and prevention of breast cancer. Int J Clin Pract. 2007;61(12):2051-2063.

13. Rodriguez-Wallberg KA, Oktay K. Fertility preservation in women with breast cancer. Clin Obstet Gynecol. 2010;53(4):753-762.

14. Smith IE, Dowsett M, Yap YS, et al. Adjuvant aromatase inhibitors for early breast cancer after chemotherapy-induced amenorrhoea: caution and suggested guidelines. J Clin Oncol. 2006;24(16):2444-2447.

15. Mouridsen H, Gershanovich M, Sun Y, et al. Superior efficacy of letrozole versus tamoxifen as first-line therapy for postmenopausal women with advanced breast cancer: results of a phase III study of the International Letrozole Breast Cancer Group. J Clin Oncol. 2001;19(10): 2596-2606.

16. Bonneterre J, Buzdar A, Nabholtz JM, et al. Anastrozole is superior to tamoxifen as first-line therapy in hormone receptor positive advanced breast carcinoma. Cancer. 2001;92(9):2247-2258.

17. Nabholtz JM, Buzdar A, Pollak M, et al. Anastrozole is superior to tamoxifen as first-line therapy for advanced breast cancer in postmenopausal women: results of a North American multicenter randomized trial. Arimidex Study Group. J Clin Oncol. 2000;18(22):3758-3767.

18. Mouridsen HT. Aromatase inhibitors in advanced breast cancer. Semin Oncol. 2004;31(6 Suppl 12):3-8.

19. Joel PB, Traish AM, Lannigan DA. Estradiol-induced phosphorylation of serine 118 in the estrogen receptor is independent of p42/p44 mitogenactivated protein kinase. $J$ Biol Chem. 1998;273(21):13317-13323.

20. Macedo LF, Sabnis G, Brodie A. Preclinical modeling of endocrine response and resistance: focus on aromatase inhibitors. Cancer. 2008; 112(3 Suppl):679-688.

21. Macedo LF, Sabnis GJ, Goloubeva OG, Brodie A. Combination of anastrozole with fulvestrant in the intratumoral aromatase xenograft model. Cancer Res. 2008;68(9):3516-3522.

22. Bergh J, Jonsson PE, Lidbrink EK, et al. FACT: an open-label randomized phase III study of fulvestrant and anastrozole in combination compared with anastrozole alone as first-line therapy for patients with receptorpositive postmenopausal breast cancer. J Clin Oncol. 2012;30(16): 1919-1925.

23. Mehta RS, Barlow WE, Albain KS, et al. Combination anastrozole and fulvestrant in metastatic breast cancer. $N$ Engl J Med. 2012;367(5): 435-444.

24. Johnston SR, Kilburn LS, Ellis P, et al. Fulvestrant plus anastrozole or placebo versus exemestane alone after progression on non-steroidal aromatase inhibitors in postmenopausal patients with hormone-receptorpositive locally advanced or metastatic breast cancer (SoFEA): a composite, multicentre, phase 3 randomised trial. Lancet Oncol. 2013;14(10): 989-998.

25. Burstein HJ, Prestrud AA, Seidenfeld J, et al. American Society of Clinical Oncology Clinical Practice Guideline: Update on Adjuvant Endocrine Therapy for Women With Hormone Receptor-Positive Breast Cancer. J Clin Oncol. 2010;28(23):3784-3796.

26. Cuzick J, Sestak I, Baum M, et al. Effect of anastrozole and tamoxifen as adjuvant treatment for early-stage breast cancer: 10-year analysis of the ATAC trial. Lancet Oncol. 2010;11(12):1135-1141.

27. BIG 1-98 Collaborative Group, Mouridsen H, Giobbie-Hurder A, et al. Letrozole therapy alone or in sequence with tamoxifen in women with breast cancer. N Engl J Med. 2009;361(8):766-776.
28. Kaufmann M, Jonat W, Hilfrich J, Eidtmann H, Gademann G, Zuna I, von Minckwitz G. Improved overall survival in postmenopausal women with early breast cancer after anastrozole initiated aftertreatment with tamoxifen compared with continued tamoxifen: the ARNO 95 Study. J Clin Oncol. 2007;25(19):2664-2670.

29. Boccardo F, Rubagotti A, Guglielmini P, Fini A, Paladini G, Mesiti M, Rinaldini et al. Switching to anastrozole versus continued tamoxifen treatment of early breast cancer. Updated results of the Italian tamoxifen anastrozole (ITA) trial. Ann Oncol. 2006;17 Suppl 7:vii10-4.

30. Coombes RC, Hall E, Gibson LJ, Paridaens R, Jassem J, Delozier $\mathrm{T}$, Jones SE, et al. Intergroup Exemestane Study. A randomized trial of exemestane after two to three years of tamoxifen therapy in postmenopausal women with primary breast cancer. $N$ Engl $J$ Med. 2004;350(11):1081-1092.

31. Jakesz R, Greil R, Gnant M, et al. Extended adjuvant therapy with anastrozole among postmenopausal breast cancer patients: results from the randomized Austrian Breast and Colorectal Cancer Study Group Trial 6a. J Natl Cancer Inst. 2007;99(24):1845-1853.

32. Goss PE, Ingle JN, Martino S, et al. Randomized trial of letrozole following tamoxifen as extended adjuvant therapy in receptor-positive breast cancer: updated findings from NCIC CTG MA.17. J Natl Cancer Inst. 2005;97(17):1262-1271.

33. Mamounas EP, Jeong JH, Wickerham DL, et al. Benefit from exemestane as extended adjuvant therapy after 5 years of adjuvant tamoxifen: intention-to-treat analysis of the National Surgical Adjuvant Breast And Bowel Project B-33 trial. J Clin Oncol. 2008;26(12):1965-1971.

34. College of American Pathologists. Breast Cancer Invasive Lobular Carcinoma. College of American Pathologists; 2010. Available from: http://www.cap.org/apps/docs/reference/myBiopsy/BreastInvasiveLobularCarcinoma.pdf. Accessed February 15, 2015.

35. Gnant M, Mlineritsch B, Schippinger W, et al. Endocrine therapy plus zoledronic acid in premenopausal breast cancer. $N$ Engl J Med. 2009; 360(7):679-691.

36. Pagani O, Regan MM, Walley BA, et al. Adjuvant exemestane with ovarian suppression in premenopausal breast cancer. N Engl J Med. 2014; 371(2):107-118.

37. Francis PA, Regan MM, Fleming GF, et al. Adjuvant Ovarian Suppression in Premenopausal Breast Cancer. N Engl J Med. 2015;372(5): 436-446.

38. Hershman DL. Perfecting Breast-Cancer Treatment - Incremental Gains and Musculoskeletal Pains. N Engl J Med. 2015;372(5):477-478.

39. Rugo HS. Hormone therapy in premenopausal women with early-stage breast cancer. N Engl J Med. 2014;371(2):175-176.

40. Waters EA, Cronin KA, Graubard BI, Han PK, Freedman AN. Prevalence of tamoxifen use for breast cancer chemoprevention among U.S. women. Cancer Epidemiol Biomarkers Prev. 2010;19(2):443-446.

41. Waters EA, McNeel TS, Stevens WM, Freedman AN. Use of tamoxifen and raloxifene for breast cancer chemoprevention in 2010. Breast Cancer Res Treat. 2012;134(2):875-880.

42. Goss PE, Ingle JN, Ales-Martinez JE, et al. Exemestane for breast-cancer prevention in postmenopausal women. $N$ Engl J Med. 2011;364(25): 2381-2391.

43. Cuzick J, Sestak I, Forbes JF, et al. Anastrozole for prevention of breast cancer in high-risk postmenopausal women (IBIS-II): an international, double-blind, randomised placebo-controlled trial. Lancet. 2014; 383(9922):1041-1048.

44. Loprinzi CL, Barton DL, Qin R. Nonestrogenic management of hot flashes. J Clin Oncol. 2011;29(29):3842-3846.

45. Niravath P. Aromatase inhibitor-induced arthralgia: a review. Ann Oncol. 2013;24(6):1443-1449.

46. Henry NL, Banerjee M, Wicha M, et al. Pilot study of duloxetine for treatment of aromatase inhibitor-associated musculoskeletal symptoms. Cancer. 2011;117(24):5469-5475. 
International Journal of Women's Health

Dovepress

\section{Publish your work in this journal}

The International Journal of Women's Health is an international, peerreviewed open-access journal publishing original research, reports, editorials, reviews and commentaries on all aspects of women's healthcare including gynecology, obstetrics, and breast cancer. The manuscript management system is completely online and includes

Submit your manuscript here: http://www.dovepress.com/international-journal-of-womens-health-journa a very quick and fair peer-review system, which is all easy to use. Visit http://www.dovepress.com/testimonials.php to read real quotes from published authors. 